\title{
Comparative Analysis of Lossless Image Compression techniques SPHIT, JPEG-LS and Data Folding
}

\author{
Mohd imran $^{1}$, Tasleem Jamal ${ }^{2}$, Misbahul Haque ${ }^{3}$, Mohd Shoaib ${ }^{4}$ \\ 1,2,3,4 Department of Computer Engineering, Aligarh Muslim University, Aligarh, India \\ ${ }^{1}$ mimran.ce@amu.ac.in \\ 2 tasleem.jamal@zhcet.ac.in \\ ${ }^{3}$ misbahul.haque@zhcet.ac.in \\ ${ }^{4}$ md.shoaibs@gmail.com
}

\begin{abstract}
Today`s era is all about Big data. The stream of data which is continuously generated by Internet requires a huge storage capacity. In spite of the availability of various data center and content delivery networks, it also imposes constraints on it. In this scenario, we require compression mechanism that can successfully reduce the bulk amount of data and also capable of perfectly reconstruct it. In this paper we have work on the restoration of compressed image with lossless feature using an existing novel method of data folding technique[1]. This approach is more feasible for continuous-tone images which comes in spatial domain by reducing the machine computational complexity and with smart compression as compression to existing techniques. The idea of data folding is followed in the way of dividing the images in rows and columns and then the column is folded iteratively followed by another row folding. And this process is repeated until we cover all rows and columns present in original one and hence we obtain a reduced sized image which has higher potency. For the effectiveness of approach we have compared with some of existing standard approaches of image compression like SPHIT and JPEG-LS.
\end{abstract}

Keywords: Compression techniques, LZW, spatial, SPHIT, data folding, DWT, JPEG-LS.

\section{INTRODUCTION}

Generally image describes about the visual opinion of certain object. In some applications, this objective can be fulfilled by only representing image regardless of their size. Image compression has a revolutionary impact in the area of transmission and data storage irrespective of the limited size of the storage. Due to importance of compression, various techniques and methods have been proposed used to store and transmit the images over the network. Another characteristics of these algorithms are that they also reduced the compression time of the process as well as it also insure the integrity of the graphics images while processing it. Since all information are now stored in cloud and can be view and downloaded through web, it makes graphics images portable so that it can be fetched with no time.

Various researches and experiments have been conducted to evaluate the efficiency of a good image compression technique. Various factors have been proposed like compression ratio, compression size, and entropy for compression technique. Compression Size is the reduction in volume of the source file after performing the compression technique. The ratio of the original file size (in bits) to the compressed image size in bits is called the compression ratio of the compression technique. Entropy defines the amount of bits which are used to represent the original image after coding is performed. Generally the entropy of an image compression algorithm is given by Shannon's formula:

$$
\text { Entropy, } H=-\sum_{i}^{n} P i \log 2 P i
$$

During the designing of a compression technique for an image, there are numerous parameter that must be taken into account for better achievement of compression efficiency and computational complexity of the processing. These parameters are more concerns as it varies from one environment to another, from one bandwidth to another.

The organization of the paper is as follows. Section II contains the description of basic image compression principals. Section III illustrate the literature works that have been carries out in past. In section IV, we have presented the proposed compression method based on data folding and compare the results with Lossless SPHIT and JPEG-LS method, followed by the results and discussions in section V. The last section presents the conclusion. 


\section{BASIC OF COMPRESSION PRINCIPAL}

The Image compression principal can be further divided into two major categories:

a) Lossless Image Compression

b) Lossy Compression

The image compression technique that allows the reconstruction of the compressed image without compromising the quality of original image falls under the lossless nature. While those techniques which may loss the quality of image after the reconstruction are called lossy compression techniques. Due to loss of information these techniques are well suited for network related operation while lossless techniques are generally employed in storage application of data. The bank records and all text based compression methods generally practice the lossless nature of compression method.

The organization of presented paper is as follows. Section II describes different nature of compression principals. All the related survey and work which is carries out on the presented image compression method is discussed in section III. Section IV, comprises the proposed method of data folding technique and comparative analysis of presented work with existing standard methods like JPEG-LS and SPHIT compression methodology followed by result and also discussion part in section $\mathrm{V}$. We rest the paper with conclusion in last.

\section{BACKGROUND}

Different application uses the compression technique in variety of domain such as linear or spatial domain and also takes input format of the file in various ways. To perform the compression, numerous algorithms have been applied depending upon the type of application. In multimedia technology, some of the common algorithm which are frequently employed in application are Huffman coding, Arithmetic coding, Lampel-Ziv and LampelZiv-Welsh coding scheme[2] for text data. For Image compression numerous models are practiced such as RGB, $\mathrm{YCbCr}$ chrominance model [3], and HSU. The compression techniques used for text, generally remove the redundancy of the characters that are appeared frequently in the source file and hence the size of file to send over transmission channel reduced proportionally. Various methods for the compression of image has been reviewed in.

\section{A. JPEG- LS}

JPEG-LS is image compression technique well known its lossless low complexity and near-lossless compression standards. It is an enhanced version of LOCO-1 method which is abbreviation of Low Complexity Lossless COmpression image standard. The compression ration produced by the LOCO-1 context model is much higher or nearly similar to other low level compression techniques such as arithmetic coding, and Huffman coding etc. The compression model which is generally used by JPEG-LS method ca be classified into two categories. The choice of compression depends upon type of encoder used.

a. Lossless: In this technique the values of $\mathrm{g} 1, \mathrm{~g} 2$ and $\mathrm{g} 3$ are 0 and in this situation, compression will run into run-length mode. In opposite situation it will run in normal model.[4]

b. Near Lossless: Another mode of operation provided by JPEG-LS is claimed as "near-lossless," in which every sample value in a reconstructed image component is guaranteed to differ from the corresponding value in the original image by up to a preset (small) amount $\delta[5]$. In fact, in the specification, the lossless mode is just a special case of near-lossless compression, with $\delta=0$.

Table 1: parameter sizes and values for JPEG-LS preset coding parameters

\begin{tabular}{|c|c|c|}
\hline Parameter & $\begin{array}{c}\text { Size } \\
\text { (bits) }\end{array}$ & Values \\
\hline L1 & 16 & 13 \\
\hline ID & 8 & 1 \\
\hline MAXVAL & 16 & 0, or $1 \leq$ MAXVAL $<2^{\text {P }}$ \\
\hline T1 & 16 & 0 , or NEAR $+1 \leq$ T1 $\leq$ MAXVAL \\
\hline T2 & 16 & 0, or T1 $\leq$ T2 $\leq$ MAXVAL \\
\hline T3 & 16 & 0, or T2 $\leq$ T3 $\leq$ MAXVAL \\
\hline RESET & 16 & 0, or $3 \leq$ RESET $\leq \max (255$, MAXVAL $)$ \\
\hline
\end{tabular}

B. SPHIT: This method is a progressive and suitable for low complexity image compressor[6]. It is very suitable for a proportional good compression ratio but only using SPHIT stand alone will not yield a higher image compression ratio. 


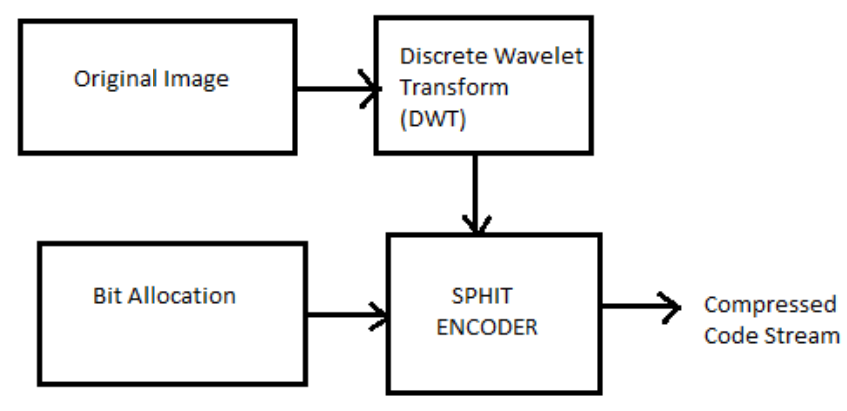

Figure 1: SPHIT Encoding Structure

SPHIT is basically a method of Set Partitioning in hierarchical trees. It is widely used in audio and video compression based on wavelet transform[7]. It is generally more preferable image compressor over JPEG-LS[8] since it is not affected by block artifact flaw as well as PSNR ratio i.e. peak signal to noise ratio curve plotted in SPHIT is high. Various improvements have been done for SPHIT to maintain the PSNR, Compression ratio as well as complexity of algorithm in

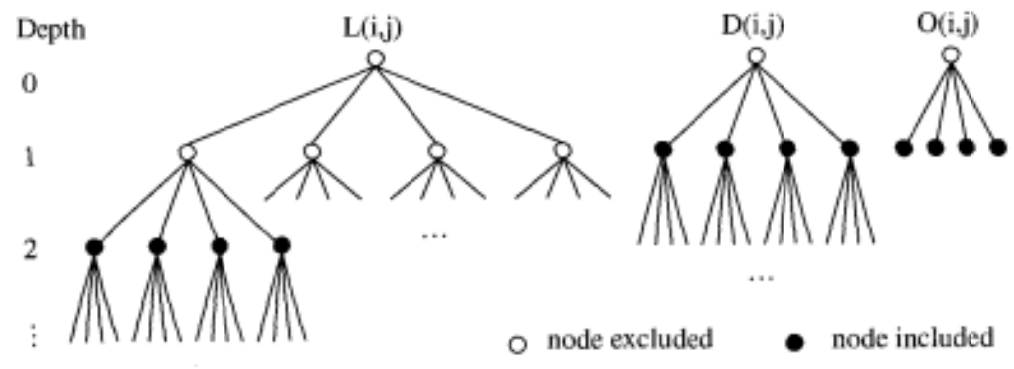

Figure 2: Types of Trees in SPHIT technique

C. Data Folding Technique:

Another strong compression technique has been developed by using data folding followed by Huffman coding scheme[1]. The key idea behind this technique is to exploit the redundancy in $2 \mathrm{D}$ image either in successive rows or columns. In this method we calculate the difference between successive even pixels of image from odd pixel and then saving it into a buffer. Now we iterate a column folding followed by a row folding step until it satisfies a necessary condition. In column folding, column adjacent pixels are subtracted while on the other hand row adjacent pixels are used for row folding. Fig3 shows the data folding technique which is followed by Huffman coding[9].

The pixel redundancies are rearranged in a tile format and source encoding technique is applied at the end before transmitting the data. The difference matrix is encoded by using the Huffman Coding (HC) algorithm. The final results are obtained after encoding the data of each level would be compressed data for the input image.

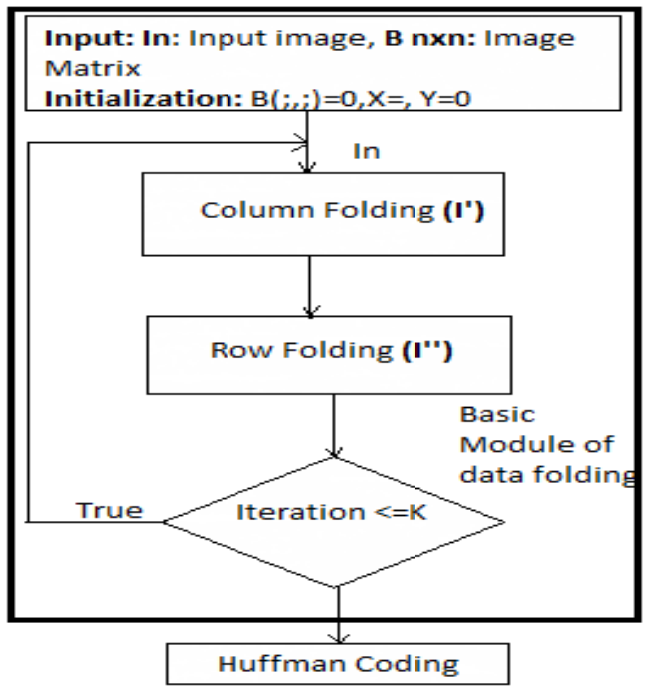

Figure 3: Flow of data in data folding technique followed by Huffman Coding 


\section{COMPARATIVE ANALYSIS OF COMPRESSION METHODS}

In this section we have taken different images and perform the compression using above said techniques. The data folding technique is followed by Huffman coding and arithmetic coding as well. Despite performing comparison among compression methods, we also have analyze compression ratio of data folding using both techniques: arithmetic as well as Huffman coding. Various parameters that have taken into account for analysis are as follows.

i. Bits Per Pixel (BPP):

BPP is used to define the no of bits required to represent the gray scale value or color value of pixel in any specified image. 24 bit/pixel give 256 possible combination of RGB (Red, Green, and Blue) information of color component in RGB model. The formula to find the Bits per Pixel is as follows:

$$
\text { Bit per Pixel, } \quad \text { bpp }=\left[\frac{\text { Size of compressed color image in bits }}{\text { No of pixels }}\right]
$$

ii. Compression ratio:

The compression ratio is very critical parameter for any image compressor which defines the how much information (redundant or irrelevant) can be reduced from source file. It is defined as the ratio of original image source file to the compressed file after performing the compression.

$$
\text { Compression ratio }=\frac{\text { Original Source image file }}{\text { File after compression }}
$$

The table consisting of images and resolution is shown in table

Table 2: Compression table for discussed algorithms

\begin{tabular}{ccccc}
\hline Image & Size & Data Folding & SPHIT & JPEG-LS \\
Image 1 & $2048 * 2048$ & 4.39 & 3.98 & 4.12 \\
Image 2 & $2048 * 2048$ & 2.04 & 1.38 & 1.12 \\
Image 3 & $2048 * 2048$ & 2 & 1.5 & 0.95 \\
Image 4 & $2048 * 2048$ & 5.68 & 4.58 & 4.37 \\
Image 5 & $1024 * 1024$ & 5 & 4.28 & 4.26 \\
Image 6 & $1024 * 1024$ & 2.84 & 1.98 & 1.86 \\
Image 7 & $4096 * 4096$ & 4.79 & 3.79 & 3.82 \\
& $512 * 512$ & 5.79 & 5.05 & 4.97 \\
\hline
\end{tabular}

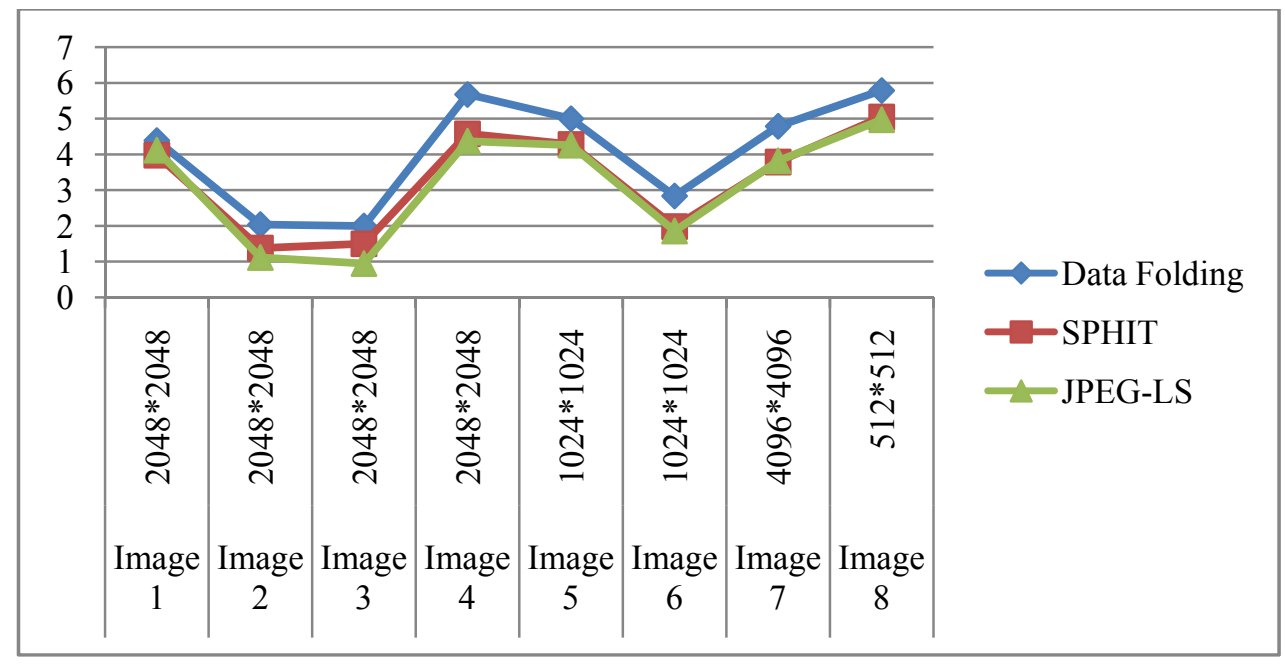

figure 4: Graph plotted between DF+HC, SPHIT and JPEG-LS Technique 


\section{RESULT AND DISCUSSION}

This section illustrate the comparative analysis of standard methods as discussed above on the various test images. As far as data folding technique is concern, we have used the Huffman coding after computing $\mathrm{k}$ iteration of successive column folding followed by row folding. To observe the compression efficiency one can replace the Huffman coding by another algorithm like arithmetic coding which further results better efficiency bit per pixel of set of images. The discussed algorithms have been applied on the taken set of test images as shown in table-2 and compression efficiency in Bit per pixel. From the curve drawn using above result it can be seen that results of each standard algorithm are relatively similar and smooth when the test image is smooth in nature.

\section{CONCLUSION}

The data folding technique is a novel method which proved a fairly good compression efficiency as well as lower computation complexity as compared to other traditional and standard algorithms which are generally used in image compression engine. It has a smooth graph and better compression ratio as it removes the redundant bits appeared in the image either in row or column which convey same amount of information. The experiment has also proved how efficient data folding technique is. Hence by seeing the comparative analysis of above SPHIT, JPEG-LS and data folding technique, we arrived at conclusion that proposed data folding technique is more promising and can be used for file as well as image compression in various network applications.

\section{REFERENCES}

[1] R. Patel, V. Kumar, V. Tyagi, and V. Asthana, "A fast and improved Image Compression technique using Huffman coding," in 2016 International Conference on Wireless Communications, Signal Processing and Networking (WiSPNET), 2016, pp. 2283-2286.

[2] M. Mozammel, H. Chowdhury, and A. Khatun, "Image Compression Using Discrete Wavelet Transform."

[3] C. Bari and T. N. Ghorude, "FACE RECOGNITION USING SKIN COLOR SEGMENTATION OF YCBCR AND RGB COLOR MODELS," vol. 23, no. 1, 2016.

[4] M. J. Weinberger, G. Seroussi, and G. Sapiro, "The LOCO-I lossless image compression algorithm: principles and standardization into JPEG-LS,” IEEE Trans. Image Process., vol. 9, no. 8, pp. 1309-1324, 2000.

[5] M. J. Weinberger, G. Seroussi, and G. Sapiro, "The LOCO-I lossless image compression algorithm: principles and standardization into JPEG-LS,” IEEE Trans. Image Process., vol. 9, no. 8, pp. 1309-1324, 2000.

[6] W. Liu, "Research on Image Compression Algorithm Based on SPHIT," in 2010 Third International Conference on Intelligent Networks and Intelligent Systems, 2010, pp. 104-107.

[7] M. Mozammel, H. Chowdhury, and A. Khatun, "Image Compression Using Discrete Wavelet Transform."

[8] V. Patil, "Review on lossless image compression using bayesian prediction combination," vol. 14, no. 2, pp. 784-787, 2015.

[9] P. A. I. Savitri, Adiwijaya, D. T. Murdiansyah, and W. Astuti, "Digital medical image compression algorithm using adaptive Huffman coding and graph based quantization based on IWT-SVD," in 2016 4th International Conference on Information and Communication Technology (ICoICT), 2016, pp. 1-5.

\section{AUTHOR PROFILE}

Mohd Imran received M.Tech. degree from Computer Engineering Department of ZH College of Engineering \& Technology of Aligarh Muslim University. He is currently working as a faculty in the Department of Computer Engineering of Aligarh Muslim University.

Tasleem Jamal received M.Tech. degree from Computer Engineering Department of ZH College of Engineering \& Technology of Aligarh Muslim University.

Misbahul Haque received M.Tech. degree from Computer Engineering Department of ZH College of Engineering \& Technology of Aligarh Muslim University. He is currently working as a faculty in the Department of Computer Engineering of Aligarh Muslim University.

Mohd Shoaib received M.Tech. degree from Computer Engineering Department of Ram Swaroop Engineering College. He is currently working as a faculty member in the Department of Computer Engineering of Aligarh Muslim University. 